\title{
Machine Vision System Measuring the Trajectory of Upper Limb Motion Applying the Matlab Software
}

\author{
Piotr Kuryło $^{1}$, Elena Pivarčiová ${ }^{2}$, Joanna Cyganiuk ${ }^{1}$, Peter Frankovský $\dot{y}^{3}$ \\ ${ }^{I}$ Institute of Machine Design and Operation, Faculty of Mechanical Engineering, University of Zielona Gora, Szafrana 4, \\ 65-516Zielona Gora,Poland,p.kurylo@ibem.uz.zgora.pl,j.cyganiuk@ibem.uz.zgora.pl \\ ${ }^{2}$ Department of Manufacturing and Automation Technology, Faculty of Environmental and Manufacturing Technology, \\ Technical University in Zvolen, Študentská 26, 96053 Zvolen, Slovakia, pivarciova@tuzvo.sk \\ ${ }^{3}$ Department of Applied Mechanics and Mechanical Engineering, Faculty of Mechanical Engineering, Technical University \\ of Kosice, Letná9,04200 Košice, Slovakia, peter.frankovsky@tuke.sk
}

\begin{abstract}
This paper discusses the problem of possibilities for applying a machine vision in the measurement of the trajectory of the upper limb movement in rehabilitation exercises. The fundamental presumption of designed system was to get the image from the camera's CCD processor, possibilities of measuring and its processing. As a result of the application system, it is possible to dynamically determine the radius between the shoulder and forearm, and also the angle between the shoulder and the chest of the man, as function of the limb motion. The created system gives the possibility to use a non-invasive method of measurement, allows visualization and full analysis of the rehabilitation progress and also allows keeping electronic records of patients.
\end{abstract}

Keywords: Machine vision system, upper limb, Matlab software.

\section{INTRODUCTION}

A typical machine vision system comprises a camera and a machine analyzing images, e.g., a computer with proper software. This kind of system must be selected with regard to the considered objectives. All the subsystems must be selected to complete the needs of a given application, and the basic issue is proper selection of the image processor in order to provide proper operation time and suitable resolution of the imagining system [1], [2], [3].

Machine vision systems are used in contactless and noninvasive measurements. These systems are applied in production processes as tools supporting different parts of these processes. They are used, among others, in quality control or in control of measurement of the geometry of products, but they are also applied in medicine [4]-[9].

Currently, the attention is mainly focused on measuring the movement of a human body in the form of a sequence of images.

The main problem related to the motion of a human body, the estimation of current configuration, is the silhouette of a human [10]. It is one of the most difficult problems in computer image processing, especially in view of diversity of human silhouettes, observation environments, and a wide range of research.
Works on observing human body motion usually use homogenous environment or simplified models [11]-[13], as well as appropriately selected clothes for a model in order to determine its characteristics. The work [14] presents a system for observing movements of a human. This system is based on a three-dimensional geometric model, taking images from three calibrated cameras. The experiment was carried out with the use of a black background and improved molecular filter with the algorithm of the simulated event, however, the obtained periods of calculation were still very long.

Multi-camera systems, built by a number of cameras [15], [16], are usually used in observing applications of human body movements (individual human) in order to obtain better results when the views of elements of human body are covered by its other elements (elements overlapping each other, thus not visible on the image). This type of application usually uses probabilistic models of the human silhouette or its three-dimensional model [11]-[13], [17].

Taking into consideration technological solutions of modern systems for motion analysis, these systems can be classified into the following groups [2], [5], [7], [9], [18]:

- Systems based on active or passive markers placed on the body of the examined person

- Ultrasonic systems 
- System based on the digitization of images obtained from cameras (few video cameras).

Systems for motion analysis enclose specialist programs for creating biomechanical models as well as for creating software [10]:

- Activation and preliminary processing of obtained data

- Preparing measurement results

- Preparing reports

Three groups can be selected from the methods of measurement used in the objective quantitative analysis of a human individually [10]:

- The first group includes methods for measuring parameters such as time, and features describing walk (the speed of walk, the frequency and length of step, etc.), that is the cycle of periodical motions.

- The second group includes kinematic methods measuring the trajectory of motion in chosen points of the body, examining motion in the three-dimensional space, as well as direct or indirect measurements of joint angles, measuring the acceleration and speed of one section of the body relevant to the adjacent section and also determining the position of individual segments of the body relevant to each other.

- The third group encloses kinematic methods for measuring forces (directly or indirectly) and moments of forces occurring in the course of the movement process.

The correct range of movement of a joint is one of the most important factors influencing efficiency; the method of measuring this range is one of the basic methods of assessing the state of the motor organ and a standard for the evaluation of the rehabilitation results. The measurement of the range of mobility in joints is carried out with the use of specialist instruments called goniometers. They can be significantly diversified: starting from simple ones, based on the principle of a standard protractor, gravitational protractor, to more complicated - electronic instruments, using the phenomenon of the change, capacity of covers, condenser depending on the location, or the change of resistance of sphygmomanometers. Simple goniometers are sufficient for static measurements of passive ranges of movements in joints. However, when the angle position should be measured in a continuous way, it is necessary to use electronic goniometers. Such situation takes place in measurements of ranges in dynamic conditions, in conditions of motion [1], [5], [6].

The measurement ranges of movements have been found to be relatively non-objective. This might result from arbitrary selection of positions of a human body in which the measurement is carried out (differences can reach even circa $20 \%$ of value) or from a relatively considerable human error due to the fact that a number of people conducted measurements. In previous years, the SFTR (S - bone surface of sagittal, F - frontal surface of sagittal, T - transverse surface of sagittal, R - rotation surface) measurement method supplied with a results recording feature has gained in popularity [18]. Its aim is to increase the scale of objectives of assessment in the process of measuring the range movements. Movements in all joints are measured from the neutral zero position, all positions and movements are described in three basic planes with use of the SFTR method.

\section{SUBJECT \& METHODS}

\section{A. Concept of machine vision system construction}

The present work illustrates the concept of construction and functions of a machine vision system that can be treated as a new method for measuring the mobility of the upper limb. The suggested method of measurement of the trajectory of upper limb movement (consequently - of any limb) is an alternative method for standard methods of measurement. It is a modern method of measuring the trajectory of upper limb movement depending on the so-called goniometry, which is the leading method of measurement on rehabilitation wards in hospitals all over the world.

The principal objective of the research was to prepare a vision system machine that would enable measuring the trajectory in rehabilitation exercises of the upper organ.

The basic assumption of the designed system was to significantly upgrade the efficiency of doctors' and/or therapists' work. The additional assumption was to create a system with possibilities of data measurement processing and provide a full visualization of all movements made by the patient in different dimensions. The full analysis, for example in $3 \mathrm{D}$, was assumed to serve a complete deep analysis of a specific case of illness. The presented elaboration shows only principal assumptions and results of the first test that was conducted on the rehabilitation ward that the authors cooperated with.

The application enabled observing the progress in the treatment of the patient. The use of this system enabled more precise establishment of a set of exercises that truly supported the improvement of joint mobility. The system allows assessing the accuracy of adequate means in the optimization of the applied treatment.

The construction of the system includes a digital photocamera with a CCD converter produced by NIKON, with the effective matrix of $6.1 \mathrm{Mpx}$, with the possibility of manual setting of lens parameters NIKIFOR. This choice allowed obtaining maximum sharpness of features, interesting from the point of view of the examination, examined person, and minimizing the factors that are not important in the analysis.

As a result of a number of analyses and trials (twenty experiments in two hospitals in Poland), to determine the position of an upper limb of the patient, the system was supplied with diodes with light intensity of $500 \mathrm{mcd}$, improved lamination, common availability, which allowed automatic instant identification of markers by the system, with the codename MAK-1.

Fig.1. shows the concept of the examination station. The construction of the system assumes conditions presented below: which are to ensure proper functions of the system:

a) Constant conditions:

-White walls,

-White screen,

- Converter equipped with CCD matrices,

- Halogen illumination.

b) Variable conditions:

- Regulation of the height distances of the CCD converter,

- Adjustment of luminance. 


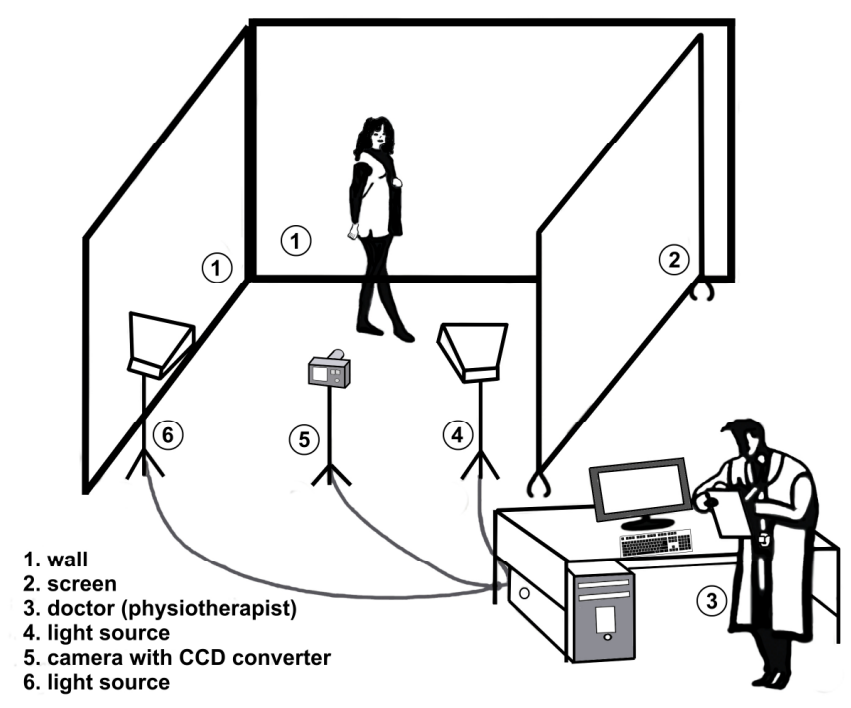

Fig.1. The concept of an examination station for the application MAK-1.

The idea of the method of measuring the localization markers (reference points) is based on the algorithm presented in Fig.2. It is an algorithm measuring the localization of markers.

The GUI operator panel defines the view of the main window of the program, the functions of operation are in other windows and buttons. This module was created with the help of the Matlab module called GUIDE [14], [18].

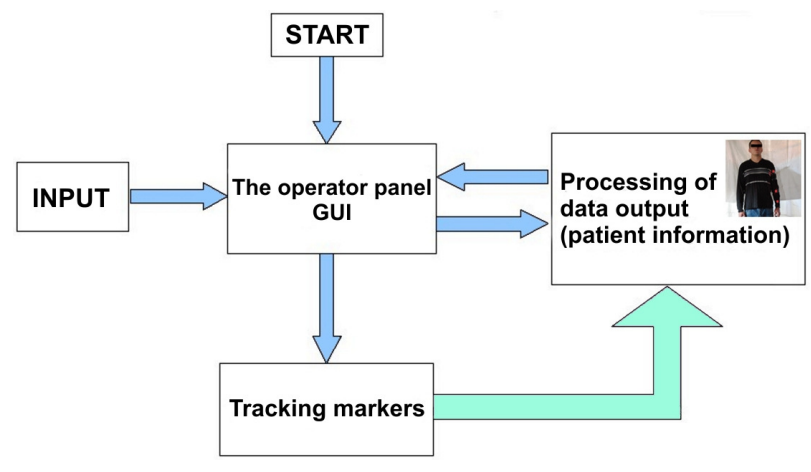

Fig.2. Algorithm measuring the localization of markers - diagram of system.

The system was built on a platform for numerical calculations in the form of a Matlab program. Matlab is an application dedicated mainly to numerical calculations and graphical presentation of obtained results. It is available for different equipment and system platforms (Windows, Macintosh, UNIX). Matlab combines an interactive software environment and a high-level programming language. The package is created mostly for scientific, technical, and engineering calculations and visualization in two or three dimensions. This program allows making complicated numerical calculations with final result presented in the form of visualization. Matlab combines numerical analysis, matrix calculations, signal processing, symbolic calculations, graphics, and creates an easy to use environment.
The standard Matlab also offers a very useful tool - GUIDE - which is an editor of the user's graphical interface used in the created system. This editor allows building windows of applications applied to standard controls.

All controls can be programmed by applied standard Matlab commands to create a program that would meet even the highest requirements. Each control has its own characteristics; therefore, it can be configured in any way needed for the application.

Matlab also gives access to numerous methods of visualization of obtained calculation results [19].

Matlab is an extended environment intended for engineering calculations creating simulations. It features a large number of functional libraries and toolboxes, possibilities are significantly enlarged by functions written by user. The programming language in the Matlab package is a rightful high-level programming language, with a syntax modelled on the $\mathrm{C}$ language. This allows using functions and structures and enables writing object oriented programs. It allows drawing two- and three-dimensional graphs of functions and visualizing results of calculations in the form of static drawing and animations [14], [20]-[23].

GUI (Graphical User Interface) is a system supporting the working of the Matlab program. It enables the user to give many commands in the same time automatically (for example by pushing an adequate button on the keyboard); it also facilitates work for inexperienced Matlab users. Designing GUI is also the first step for creating individual applications.

In point of characteristic view presented above, the prepared system realizes three fundamental blocks of operations:

- Initialization of the GUI and entering the input data (identification data of the patient),

- Observation of markers,

- Processing output data (like localization of symbols in the system of coordinates and determining particular angles between the arm and the chest).

In module "Initiation of GUI", all GUI functions are configured, i.e. functions defining the appearance of the main screen of the program, as well as functions for operating the windows and buttons. This module was created with the use of a Matlab module, called GUIDE.

In module "Input data entering", a photograph which will further be processes being loaded, and also all variables of the program, are initiated: the measure of the elbow angle, the measured value of the angle between the arm and the chest, the table recorded localizations of markers, etc. [24]-[27].

\section{B. Observation of markers}

It is the main calculation procedure of the program. It is responsible for detecting the position of markers. The principle used in the process of detecting markers is as follows: the loaded photo is scanned in a loop, pixel by pixel (in our case the scanning made from the left side to the right side, along vertical lines, from their tops to their bottoms). 
After selecting the value of the pixel - it encloses three values (R, G, B), where R, G, and B are components of color, which take values from 0 to 255 ; then we check whether the pixel is red. In our case, we accepted the following criterion: a pixel is red when its coordinate $R$ is bigger than 200 and other coordinates are smaller than 100. A pixel that meets the presented criterion is a candidate for searching the marker. The marker shown in the photograph is not represented by only one pixel, but by the entire group of pixels, therefore, it is a moment to decide whether it is worth to store these newly found coordinates. For that purpose, a procedure (Put to the list) is used. The procedure is aimed at verifying if the list of stored found positions includes a pixel localized near to the analyzed one. This process involves making calculations of the distance between pixels and stating whether their distance is big or small. In our case the distance was too big and was excluded, it is assumed to be 25 pixels - point markers (number of pixels is determined by technical possibilities of the camera used in the measuring process). The distance between the patient and the camera was constant and amounted to $3 \mathrm{~m}$. The distance was established experimentally. The diameter of the used diodes was $5 \mathrm{~mm}$. After observing all pixels in the picture, coordinates of pixels were recalculated in a standard system of coordinates, with its beginning in the left-bottom (not upper) corner and their coordinates were ordered in a growing sequence on the $x$ axis.

The procedure of the search for markers seems to be every time-consuming, therefore, it is better to decompose the entire image into several smaller ones and search the solution in the form of a tree.

The scheme of operation of the main calculation is shown in Fig.3.

\section{Output data processing}

After detecting the locations of markers, applied to a tested body, and appropriate transformation of their coordinates, the angle between vectors determined by particular pixels is calculated. The formula (1) was used for establishing the angle $\varphi$ between two vectors $\mathrm{v} 1$, v2:

$$
\cos \varphi_{\mathrm{v} 1, \mathrm{v} 2}=\frac{\mathrm{v} 1 . \mathrm{v} 2}{|\mathrm{v} 1||\mathrm{v} 2|}
$$

where $\mathrm{v} 1, \mathrm{v} 2$ are a standard scalar product, i.e. the sum of products of subsequent coordinates and $|\mathrm{v} 1|,|\mathrm{v} 2|$ are the vector sizes. Next, after obtaining the cosine of the required angle, applied function inverse to the cosine function Matlab - as cosine function, the measure of the angle is established.

Vectors v1 and v2 (Fig.4.) are defined by positions of markers that are located on the tested person's clothes, read by digital camera and located by software equipment.

After calculating the value of angles, all values are presented in suitable GUI windows. It is possible here to make visualization of last localizations of markers, five sets of localizations of these markers, too.

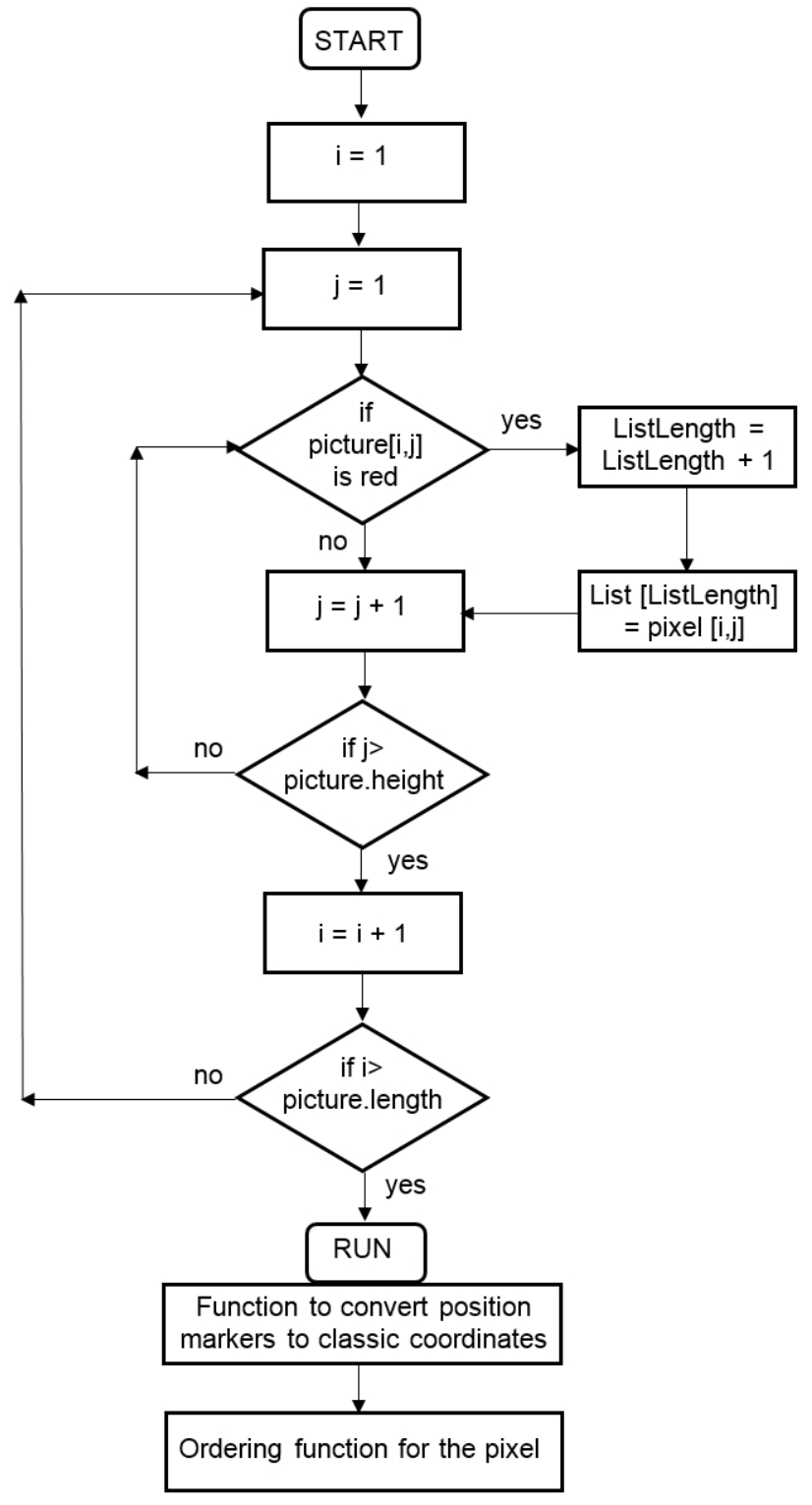

Fig.3. Scheme of the main numerical procedure.

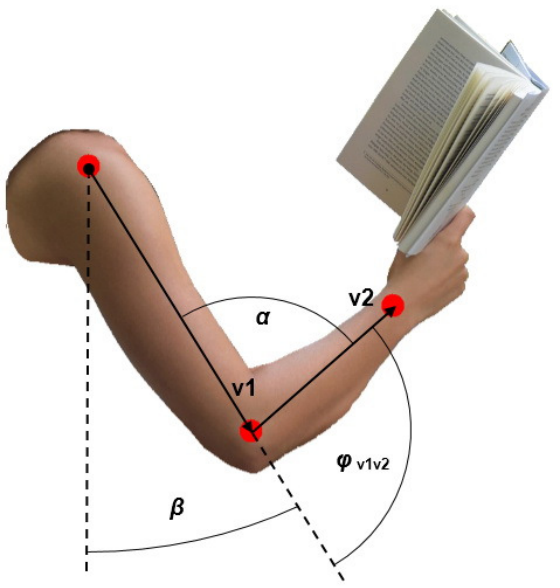

Fig.4. Presentation of $\varphi$ angle between vectors v1 and v2, defined by the markers' position. 


\section{Hardware elements}

The hardware of the system includes the following elements:

- One-objective apparatus - digital reflex camera with the matrix CCD with 6.1 million effective pixels Nikon D40 with a NIKIFOR objective, with option of manual placing of the screen and the focal length,

- Photographs which are made in the JPEG form (the standard size of photographs is: $3008 \times 2000$ pixels, scaled into the format $640 \times 480$ in order to make the analysis of the image faster),

- The computer with the Matlab software.

Fig.5. represents the design of an operator's panel for the system MAK-1, where: 1 - loading the image, 2 - button enabling to detect markers; 3 - button resetting (cancelling) current commands; 4 - coordinates of markers' localization $(x, y) ; 5$ - the $\alpha$ angle (the angle between the arm and the forearm - angle was measured counter clockwise); 6 - the $\beta$ angle (angle between the arm and the torso - angle was measured counter clockwise); 7 - button enabling reading the last localizations of markers; 8 - for storing localizations of markers, 9 - button enabling drawing the graph (the trajectory of the movement of a limb - after choosing the button no. 8); 10 - button for closing the application.

Function no. 8 gives the possibility to add new graphs to the already existing ones; and this facilitates assessing the range of limb's movements.

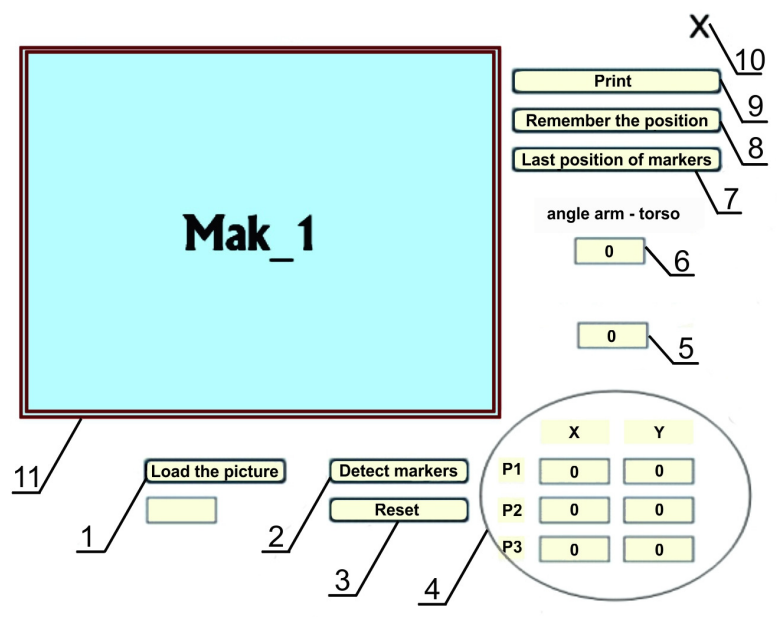

Fig.5. Illustration presented operator's panel in application MAK-1.

\section{CALiBRATION OF THE MAK-1 SYSTEM}

Calibration of the vision system was carried out based on the single camera method (monovision) by using the Matlab Camera Calibration Toolbox. The Toolbox allows to measure the distance between three points (three points corresponding to the position of measurement technique for three characteristic points on the upper limb of the patient).

To obtain information about the location of measurement points, it was necessary to determine the coordinates $(x, y)$. Then information about the location of these points in the space $(x, y)$ was obtained.
The calibration through usage of Matlab Camera Calibration Toolbox required 20 images. It was important that all measuring points were present in each image.

Fig.6. presents points $\mathrm{P}_{1}, \mathrm{P}_{2}, \mathrm{P}_{3}$ that represent the three measured points, mapping the distance between these points in two-dimensional space.

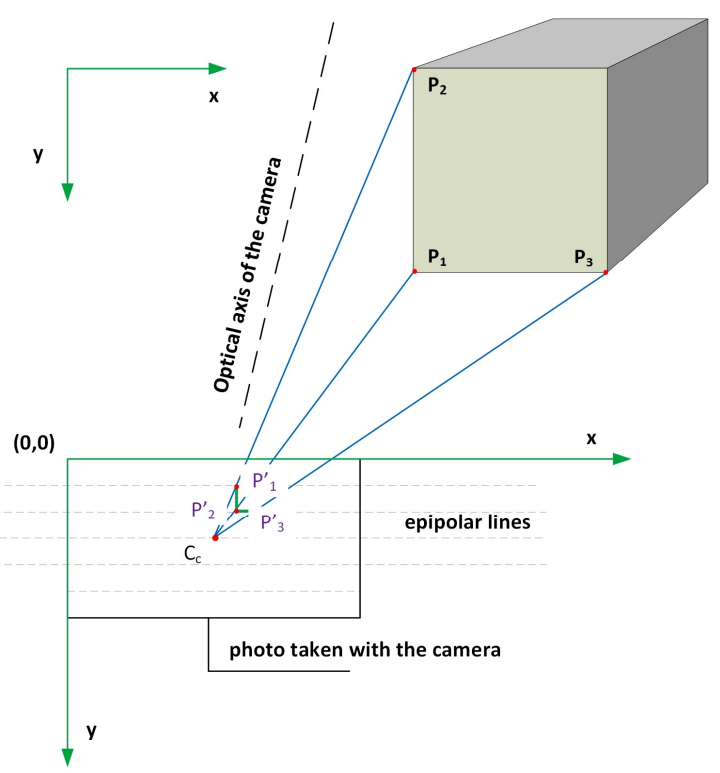

Fig.6. Calibration method of the MAK-1 system by using NIKON 40 camera.

In the canonical system, the corresponding points in image plane of the camera are connected by so-called peripolar lines.

Based on 20 pictures prepared by monovision technique, it was necessary to properly perform the calibration procedure, verification of the distance between measuring points using the Camera Calibration Toolbox in Matlab package. The average error of an image reprojection was 0.8 pixels per photo.

\section{RESUlts}

Fig.7. presents the examined movement in the frontal plane for three angle deflections.

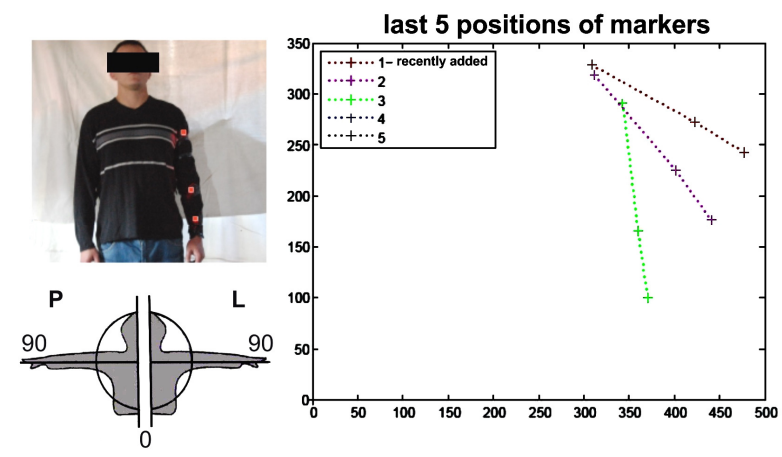

Fig.7. Movement of trajectory for three angle deflections in the examination of upper limb abduction. 
Fig.7. presents the results of measurements of trajectories of position measured points in frontal position of the patient. In order to determine the patient's movement abilities in abduction of the upper limb, the position of markers was recorded in frontal position. Curve 1 presents the maximum abduction of upper limb after effective rehabilitation. Curve 2 shows the position of markers during the maximum deflection of patient's upper limb before rehabilitation. Curve 3 presents the position of markers in the resting position of the patient's arm before rehabilitation.

The examined angle deflections of the elbow are presented in Fig.8. It is a screenshot from MAK-1 application. The trajectories present the elbow joint movements for five measurements.

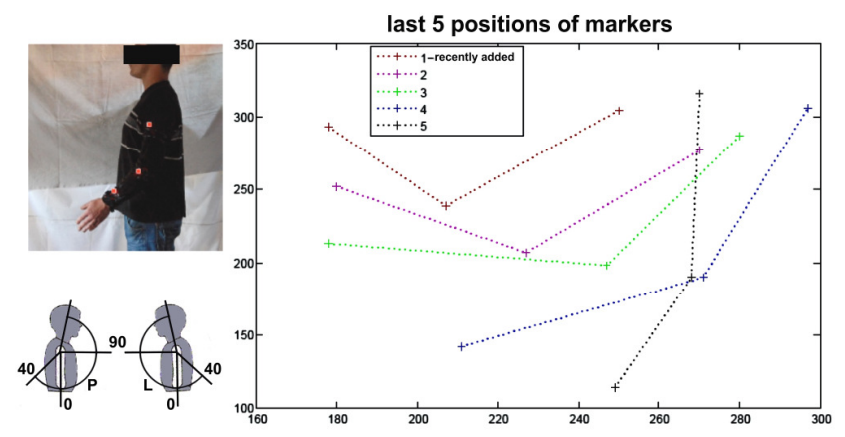

Fig.8. Movement of trajectory for five angle deflections in the examination of the elbow joint in the upper limb.

It is important that the progress in process of deflecting a segment of the forearm (in reference to the arm) may be observed, which will help to give clear image in the case of long lasting rehabilitation, for instance after a severe accident. A doctor whom controls the treatment has the possibility to accept a suggested set of exercises or he/she could reject it entirely. $\mathrm{He} / \mathrm{she}$ can also suggest an additional set of exercises, in order to obtain better health improvements of the treated patient.

The examination of bend and extension of the upper limb in the transverse plane can be seen in Fig.9. The figure presents a screenshot from the MAK-1 application.

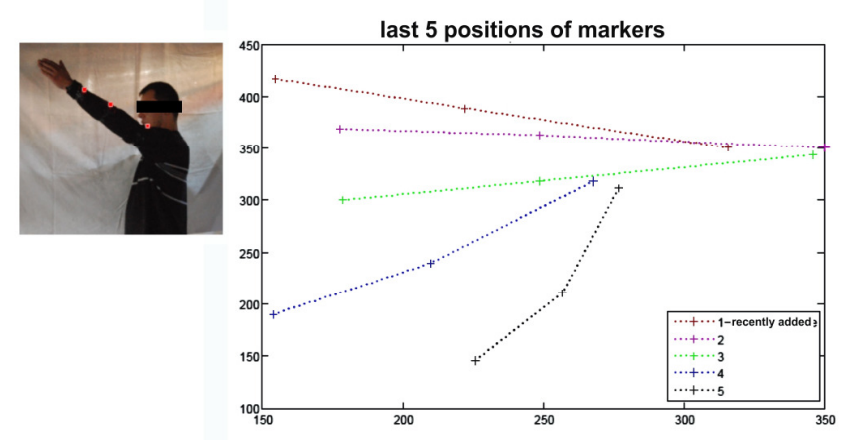

Fig.9. Trajectory for five angle deflections in the range of movements in the shoulder joint.
The Fig.8. present results of the trajectories of measured points in sagittal position of mobility of the elbow joint. In the past, to determine the patient's movement abilities in abduction of the upper limb, the position of the markers was recorded in sagittal position. Curve 1 presents the maximum abduction of rehabilitated upper limb after introduction of effective rehabilitation. Curves 2-3 present the location of markers during rehabilitation. Curve 4 presents positions of markers for maximum possible deflection of the upper limb before its rehabilitation. Curve 5 shows locations of markers in the resting position of upper limb before its rehabilitation.

In Fig.9. results of measurements of trajectories of measured points in sagittal position are presented - mobility of shoulder joint. In order to determine the patient's movement abilities in abduction of the upper limb, location of markers are recorded in sagittal position. Curve 1 presents the maximum abduction of rehabilitated upper limb after introduction of effective rehabilitation. Curves 2-3 present the location of markers during rehabilitation. Curve 4 presents positions of markers for maximum possible deflection of upper limb before its rehabilitation. Curve 5 shows locations of markers in resting position of the upper limb before its rehabilitation.

The form of graphs presented in Fig.7., Fig.8., Fig.9. was consulted and accepted by orthopedists and physiotherapists. The trajectories (points in graphs) for the doctor who was caring for the patient are the most important parameter to determining his/her possibility of movement and progress in the rehabilitation process. Performance mobility of patient in the form of movements of markers as a polynomial function was difficult for interpretation for doctors and physiotherapists. Results of measurement presented as a polynomial function increased the time of diagnosis, increased the time of interpretation of assessment, and the efficiency of the patient.

\section{CONCLUSIONS}

The evolution of computing technology is progressing hand in hand with the evolution of other natural, technical or economic sciences. Adequate powerful computing technology enables high precision analysis of process models that more precisely describe the physical or social nature of things [29].

Although vision-based methods for human motion analysis have caught much attention of researchers and practitioners involved in gaming, security and other related applications, the robustness of the systems is far from ideal [28]. Over the years, physical rehabilitation has seen its relevance grow fast, drawing a lot of attention from engineering since improving the quality of life today has a higher impact as population ages. The ultimate goal of rehabilitation process should be to fully recover people from temporary motor impairments, or, in case of permanent disorder, at least to mitigate patients' struggles by aiming at as high a level of independence as possible [30].

A system for measuring the range of mobility of the upper limb was created within the frames of the realized research. The system allows determining the trajectory of the examined limb in the examined plane of motion. The system can keep 
records up to five deflections of the upper limb; therefore, it allows making a comparative analysis for several examinations. However, it is possible to determine the value of the angle between the arm and the torso automatically. This constitutes a practical determinant for the examination of the shoulder joint. Due to complexity and appropriation in life, the shoulder joint is one of the fundamental motoric organs of the human body. The MAK-1 system can function with the assumption that the examined limb has to have three marked reference points, i.e. so-called markers. They will be automatically observed during the examination; then the markers will be archived and converted to a standard coordinate system, in order to calculate the required angles $(\alpha$ and $\beta$ ).

During experiments with patients, the NIKON D40 camera was used. The necessary time for the camera to capture one position was 0.1 second. The time of recording of all measured points was 0.5 seconds. The total time of registration, measurement and determining of the trajectory between points and their visualization on PC screen was 5 seconds. The measurement was carried out in real time.

The idea of application was realized to allow its further development and adjustment to other tasks, for example to examine the lower limb, to examine the range of the limb mobility on a workstation of an industrial machine operator, etc.

One problem that occurred while implementing the presented system was to define the reference points. Luminescence diodes (markers) with an improved level of luminescence were used for this purpose; they were placed on the body of the examined person by means of a self-adhesive Velcro tape. The use of this tape can be risky, because it might cause the change of location of the utilized reference points, which might affect the reliability and unambiguity of the research results. The further stage of the research should consider the possibility of using fluorescent markers that could be placed directly on the object. First attempt was made by applying a marker produced by ORION-KAMET Company but results were not satisfactory because not all markers were detected. This suggests that the problem might be solved by application of suitable illumination or by other methods for object identification. The next step for developing the system should combine the application MAK1 and the application of the decision-making system that would determine more precisely the type of examination, the selection of mobility plane and a determined orientation of markers on this plane. The last considered change will involve supplying the system with a decisive function of recording the patients' data since according to the Act on the Protection of Personal Data the data should be encoded.

\section{ACKNOWLEDGMENT}

This work was supported in part by the Ministry of Education of the Slovak Republic Foundation under grant projects VEGA No. 1/0731/16 and VEGA No. 1/0290/18 and by project KEGA MŠ SR 003TU Z-4/2016: Research and education laboratory for robotics and APVV-17-0258 Digital engineering elements application in innovation and optimization of production flows and from the program of the Polish Minister of Science and Higher Education 003/RID/2018/19: Regional Initiative of Excellence.

\section{REFERENCES}

[1] Meyer-Baese, A. (2004). Pattern Recognition for Medical Imaging. Academic Press.

[2] Russ, J.C. (2007). The Image Processing Handbook. CRC Press.

[3] Woźnicki, J. (1996). Podstawowe techniki przetwarzania obrazu. Warsaw, Poland: Wydawnictwo Komunikacji i Łączności (Transport and Communication Publishers). (in Polish)

[4] Poppe, R. (2007). Vision-based human motion analysis: An overview. Computer Vision and Image Understanding, 108 (1-2), 4-18.

[5] Najarian, K., Splinter, R. (2005). Biomedical Signal and Image Processing. CRC Press.

[6] Rangayyan, R.M. (2004). Biomedical Image Analysis. CRC press.

[7] Acton, S., Ray, N. (2005). Biomedical Image Analysis: Tracking. Morgan \& Claypool Publishers.

[8] Tadeusiewicz, R., Ogiela, M. (2008). Modern Computational Intelligence Methods for the Interpretation of Medical Images. Springer.

[9] Nagesh, Y., Bleakley, C., Lennon, O. (2010). Wearable absolute 6 DOF exercise training system for post stroke rehabilitation. In Proceedings of the Fourth Irish Human Computer Interaction Conference. Dublin City University.

[10] Kuryło, P., Cyganiuk, J., Tertel, E., Frankovský, P. (2016). Machine vision investigate the trajectory of the motion human body - review of the methods. Acta Mechatronica, 1 (2), 7-13.

[11] Deutscher, J., Blake, A., Reid, I. (2000). Articulated body motion capture by annealed particle filtering. In Proceedings of IEEE Conference on Computer Vision and Pattern Recognition. IEEE, 126-133.

[12] Schmidt, J., Fritsch, J., Kwolek, B. (2006). Kernel particle filter for real-time $3 \mathrm{D}$ body tracking in monocular color images. In Proceedings of IEEE Conference on Automatic Face and Gesture Recognition. IEEE, 567-572.

[13] Ivekovič, Š., Trucco, E., Petillot, Y.R. (2008). Human body pose estimation with particle swarm optimisation. Evolutionary Computation, 16 (4), 509-528.

[14] Zalewski, A., Cegiełka, R. (1999). Matlab - Obliczenia numeryczne $i$ ich zastosowanie. Warsaw, Poland: Scientific and Technical Publishers. (in Polish)

[15] Rosales, R., Siddiqui, M., Alon, J., Sclaroff, S. (2001). Estimating 3D body pose using uncalibrated cameras. In Proceedings in IEEE Conference on Computer Vision and Pattern Recognition. IEEE, 821-827.

[16] Sigal, L., Bhatia, S., Roth, S., Black, M.J., Isard, M. (2004). Tracking loose-limbed people. In Proceedings of IEEE Conference on Computer Vision and Pattern Recognition. IEEE, 15-48.

[17] Sidenbladh, H., Black, M., Fleet, D. (2000). Stochastic tracking of 3D human figures using 2D image motion. 
In Proceedings of Computer Vision - ECCV $2000: \sigma^{\text {th }}$ Europen Conference on Computer Vision. Springer, 702-718.

[18] Szczechowicz, J. (2011). Pomiary katowe zakresu ruchu: zapisy pomiarów, metoda SFTR. Krakow, Poland: University School of Physical Education in Krakow. (in Polish)

[19] Wróbel, Z., Koprowski, R. (2004). Praktyka przetwarzania obrazów $w$ programie Matlab. Akademicka Oficyna Wydawnicza EXIT. (in Polish)

[20] Tomczak, L., Mosorov, V., Sankowski, D., Nowakowski, J. (2007). Image defect detection methods for visual inspection systems. In 9th International Conference - The Experience of Designing and Applications of CAD Systems in Microelectronics. IEEE, 454-456.

[21] Liu, J., Wang, X. (2012). Advanced Sliding Mode Control for Mechanical Systems: Design, Analysis and MATLAB Simulation. Springer.

[22] Shaoqiang, Y., Zhong, L., Xingshan, L. (2008). Modeling and simulation of robot based on Matlab/SimMechanics. In 27th Chinese Control Conference. IEEE, 161-165.

[23] Ostertagová, E. (2012). Aplikovaná štatistika v počitačovom prostredi MATLABu. Košice, Slovakia: Equilibria. (in Slovak)

[24] Sudharsan, J., Karunamoorthy, L. (2016). Path Planning and co-simulation control of 8 DOF anthropomorphic robotic arm. International Journal of Simulation Modelling, 15 (2), 302-312.

[25] Cuesta, E., Mantaras, D.A., Luque, P., Alvarez, B.J., Muina, D. (2015). Dynamic deformations in coordinate measuring arms using virtual simulation. International Journal of Simulation Modelling, 14 (4), 609-620.

[26] Russ, J.C., Matey, J.R., Mallinckrodt, A.J., McKay, S. (1994). The image processing handbook. Computers in Physics, 8 (2), 177-178.

[27] Lozhkin, A., Abramov, I., Bozek, P., Nikitin, Y. (2014). The issue of calculating elliptic trajectories. Manufacturing Technology, 14 (4), 561-566.

[28] Zheng, F., Shao, L., Racic, V., Brownjohn, J. (2016). Measuring human-induced vibrations of civil engineering structures via vision-based motion tracking. Measurement, 83, 44-56.

[29] Hart'anský, R., Smieško, V., Rafaj, M. (2017). Modifying and accelerating the method of moments calculation. Computing and Informatics, 36 (3), 664682.

[30] Daponte, P., De Vito, L., Riccio, M., Sementa, C. (2014). Design and validation of a motion-tracking system for ROM measurements in home rehabilitation. Measurement, 55, 82-96.

Received May 18, 2018 Accepted December 22, 2018 